\title{
Electrical Energy Harvesting from Cooker's Wasted Heat with Using Conduction Cooling
}

\author{
Wincent, Ghafour Amouzard Mahdiraji ${ }^{1, *}$ \\ School of Engineering, Taylor's University, 47500, Subang Jaya, Selangor, Malaysia
}

\begin{abstract}
In order meet the demand of electricity in current era, the need for new sources of energy even in very minimal amount, could be done with proper research and technology advancement in order to convert as much wasted energy as possible. Collecting and analyses cooker's wasted heat as a main wasted energy source become the main interest for this research. This application can be installed either in household usage or commercial usage. Based on majority stove in household datasheet it shown that the efficiency of the stove is approximately $50 \%$. With half of the efficiency turn into wasted heat, this application is suitable for thermoelectric generator (TEG) to harvest the heat. The objective of this research is to determine whether the thermoelectric generator (TEG) would able to power the 3V LED light as a small lighting system in household. Several designs with five TEGs in series circuit are tested to the application to analyses which method generated a better result. Since this research only focus in using a conduction cooling, aluminum heat sink will be utilized either for heat absorption or heat rejection. The maximum temperature differences between hot side and cold side is $209.83{ }^{\circ} \mathrm{C}$ with average power approximately $0.1 \mathrm{~W}$.
\end{abstract}

\section{Introduction}

In this contemporary society, energy demand has become one of the crucial challenge. Based on Energy Information Administration, the demand of energy in the society is annually increase without any sight of peaking. Thus, this challenge has become one of the popular topic to discuss and to motivate the society to increase the energy supply. A lot of efforts is made by researchers to meet the demand of the energy by considering either other source of energy or renewable energy. An improvement has been done by other extensive research on renewable energy such as wind turbine, tidal energy, and solar power to supply the energy demand and, yet it still could not entirely cover the demand. Therefore, new methods to gain an extra energy is developed and one of the method is to absorb the wasted heat produce in daily household appliances.

Thermoelectric energy is known to be the usable source of electricity from the conservation of wasted heat and this energy is operate based on Peltier and Seebeck effecting. In year 1821, German physicist Thomas Johann Seebeck discover that a temperature differences is existed between two dissimilar metals, electricity will be

\footnotetext{
${ }^{1}$ Corresponding author: Ghafour.AmouzadMahdiraji@taylors.edu.my
} 
generated since the electron level in each material shifted differently. Electron from the surface that is supplied with enough heat will eventually jump to the metal with colder surface. Thus, the movement of the electron is known to generate the thermoelectric energy $[1]$.

Thermal energy is commonly being classify as a by-product from various forms of energy such as mechanical energy, chemical energy and electrical energy. It is known that, machinery such as washing machine, refrigerator, cooker is used daily by the people either in household or commercial. With the usage of the machinery, the electrical energy that supplied it will generated a lot of wasted heat due to the moving part of the machine or the heat loss due to the appliances product efficiency. Therefore, this wasted heat energy contain wasted efficiency percentage that can be absorb and covert to the usable electrical energy [2].

Due to the increasing of the energy demand, research is done to find the new method to discover renewable and available energy. As such, by absorb the wasted heat from the machinery heat loss and convert them to usable electrical energy. To perform this research, Thermoelectric generator (TEG) is become one of the solution. This technology is initially used to convert the geothermal energy to the electrical energy.

Although a thermocouple will be the first thing that comes to mind when thermal energy is mentioned, a TEG cannot be related to thermocouple. This is because both devices have a different usage purpose. Thermocouple is temperature gauge device which only measures the temperature in one space, while thermoelectric generator is used to covert the heat energy to usable electricity that depend on the temperature differences between two surfaces [3].

Thermoelectric technology is a rather new, developing technology that is inexpensive to build and is space saving due to their small size as shown in Figure 1. It is also easy to use. In addition, this technology is sustainable as well as it is eco-friendly and does not produce greenhouse gases or sound pollution. A downside to this technology is its low efficiency when generating electricity, which is of approximately $5-10 \%$ of the total heat energy [4][5].

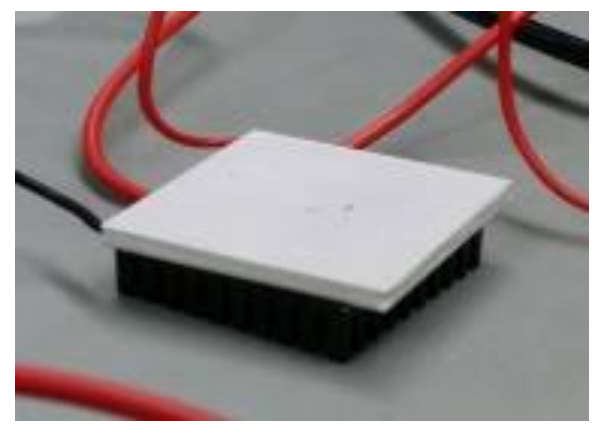

Fig. 1. Thermoelectric Generator.

The concept behind kitchen appliances, such as a cooker is studied in order to understand the heat transfer from the cooker's flame and to discover the most optimum location to install the thermoelectric generator. Efficiency and total power generated from each design will be calculated together with the total cost. This is to determine whether this application would be a success.

In year 2005, researcher form Xiamen University has done and experiment with applying same TEG material Bismuth telluride with antimony doping to the usage on the 
plantation machine. During its experiment it found that the temperature difference that produce by the machine heat with water cooling technique as heat rejection is approximately $77^{\circ} \mathrm{C}$. this produce the ZT value as big as 1.15 and voltage generated is less than $0.5 \mathrm{~V}[6]$.

By reducing the thermal conductivity, it may enhance the ZT. Pei He Jiangqing, a Student from School of material science and engineering in Beihang University, found that Ca-doped $\mathrm{BiCuSeO}$ material exhibits low thermal conductivity and resulted in increasing the ZT by 0.9 at $923 \mathrm{~K}$ [7]. Meanwhile, Zhao et al. from Northwestern University reported that SnSe single crystal that measured along with room temperature orthorhombic unit cell $\mathrm{b}$ axis produced the $\mathrm{ZT}$ of 2.6 at $923 \mathrm{~K}$ [8].

\section{Research methodology}

The method in conducting this research is discusses in this section. The research that is conducted is a quantitative study based on the simplest and most effective method. TEG has been by so many researchers especially for energy experts. Several types of thermoelectric modules have been utilised and fabricated. However, in this study only Bi2Te3 thermoelectric module is used due to its reliability and capability to produce the higher ZT among other type in room temperature. Moreover, the availability of Bi2Te3 thermoelectric module is common in the market. Although thermoelectric generator is easy to operate, multiple experiments are required to find the most effective method [9].

FYP1 project will focus on studying literature review for both thermoelectric module and cookers from either journal or online to have a better understanding on what it will dealing with and what the device are. This includes executing initial experiment two of designs and find the best location to install the TEG, and lastly is purchase the suitable equipment. ANSYS software will be used to find the best location to install the TEG. For the first design (Design A) the TEG is attach to the acrylic and hanged near the heat source which is approximately $5 \mathrm{~cm}$. small heatsink is place at the cold side in order to release the heat from the hot side. This design has a convection heat transfer thus the temperature obtained was lower compare to the other design. For second design TEG (Design B) will be place to the aluminium plate which design to have a hole at the left, this is to allow the fire is able to go through to heat the pot/pan. To place the TEG, ANSYS computer software is utilized to find the best location, and at the purple line as show in Figure 2 which is approximately $10 \mathrm{~cm}$ from the heat source was selected in order to prevent the TEG overheat easily conduction heat transfer was applied in this design. Both, the simulation and the two experiments will be done in implement stage where initial experiment is conducted. Result of the experiment will be analysed and both method will be compared. 


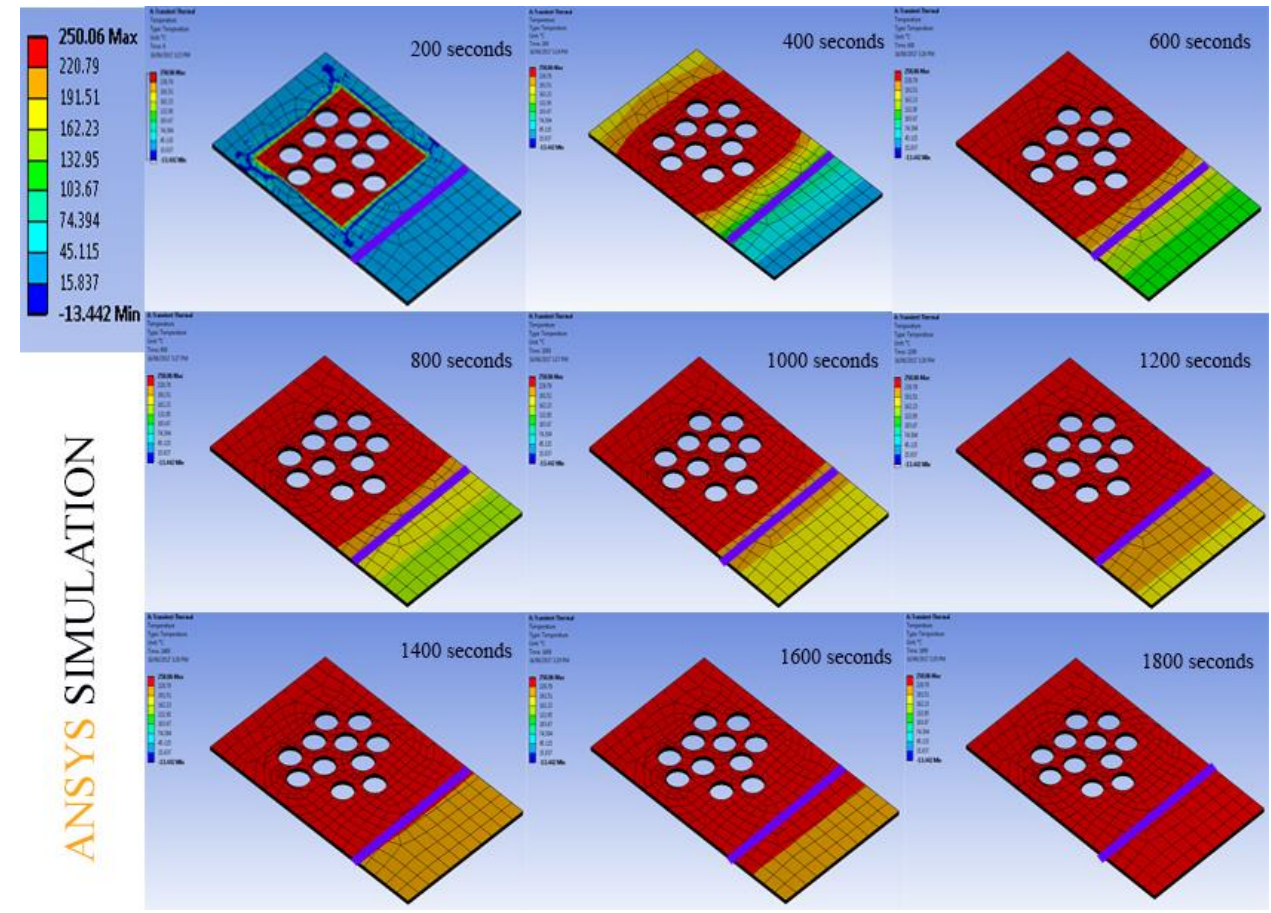

Fig. 2. ANSYS simulation for aluminium plate.

Meanwhile, to observe the differences of the result, another three Designs which are convection heat transfer and two calcium silicate with TEG facing aluminium plate (Design C), convection heat transfer and single calcium silicate with TEG facing heat source (Design D) and Design E, convection and conduction with TEG attach with L aluminium plate are tested for the experiment. Not only new designs are proposed, but high performance $\mathrm{Bi} 2 \mathrm{Te} 3 \mathrm{TEG}$, calcium silicate $\mathrm{Ca} 2 \mathrm{SiO} 4$ and big aluminium heat sink also introduce to the experiment since the old TEG performance is consider low and inefficient, the calcium silicate is to prevent the heat to transfer to the cold side and heat sink is to reject more heat to generate large temperature differences. However, with using new high performance TEG, the experiment is only conducted with using single TEG instead of using multiple TEG that form into series circuit. This is done to check whether the TEG is efficient to use before purchase multiple of them. Figure 7 show that the visual experiment setup for design $\mathrm{C}$, in this design the calcium silicate that place near to the heat source is aims to reduce the convection heat transfer to the heatsink so that the temperature differences is larger. While the calcium silicate in the bottom of TEG is aims to prevent the TEG to experience over heat since the maximum temperature of high performance TEG can withstand is $300{ }^{\circ} \mathrm{C}$ while the aluminium plate itself has temperature of $270{ }^{\circ} \mathrm{C}$ by 
using infrared thermometer gun to measure. Small heatsink is attach to the hot side which is inside the calcium silicate in order to absorb more heat and big heat sink is attached to the cold side to maximize the heat rejection. This design place near the edge of the aluminium edge which is $20 \mathrm{~cm}$ from the heat source due to consideration of the convection heat transfer to the big heatsink. Since the hot side is cover by calcium silicate, temperature of the calcium silicate will become the temperature for the hot side.

While, Design D is designs to place the TEG facing the heat source with 4 differences distance which are $15 \mathrm{~cm}, 10 \mathrm{~cm}, 5 \mathrm{~cm}$ and $2.5 \mathrm{~cm}$ from the heat source. calcium silicate is used to cover and protect the TEG and to prevent the heat to transfer to the aluminium heatsink which will be affecting the result. Small heatsink is attach to the hot side to absorb maximum heat from the heat source while big aluminium is attach to the cold side to reject the heat. In order to obtain a perfect connection, thermal grease is applied on both hot side and cold side. In addition, aluminium plate is placed to produce a better heat transfer by convection. 4 experiments are conducted and compare to each other to analyse the changes of generating voltage.

Lastly, Design E as shown in Figure 3 is conducted by placing one end of the aluminium and steel $\mathrm{L}$ bracket to attach to hot side of the TEG and the other end to fire, this is done so that conduction heat transfer is occurred and to maximize the heat absorption. Meanwhile same as the previous design, big heat sink is placed at the cold side to reject the heat and aluminium plate is placed to ensure the convection heat transfer is maximized. The results of both L bracket's material are compare. Since aluminium have a better heat conductivity with $209.5 \mathrm{~W} / \mathrm{m} . \mathrm{K}$, three differences size of aluminium L bracket $30 \mathrm{~cm}, 20$ $\mathrm{cm}$ and $15 \mathrm{~cm}$ (Figure 4) are tested and compared. Again, the best results among three differences size of $L$ bracket is tested with four difference resistors which are $10 \Omega, 7.5 \Omega$, $2.2 \Omega$ and $1 \Omega$. This is to find the power produce from the single high performance TEG. The reason several resistors are tested is to observe the behaviour of the TEG. 


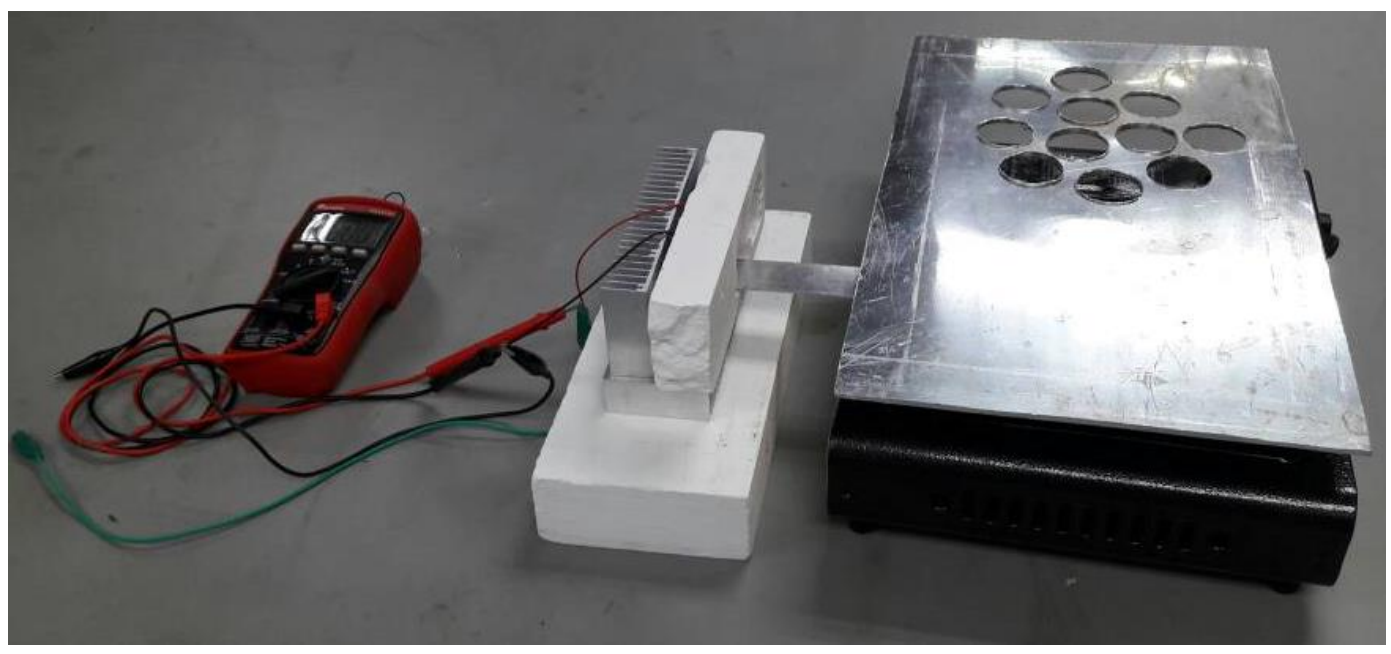

Fig. 3. $20 \mathrm{~cm}$ aluminium $\mathrm{L}$ bracket experiment setup.

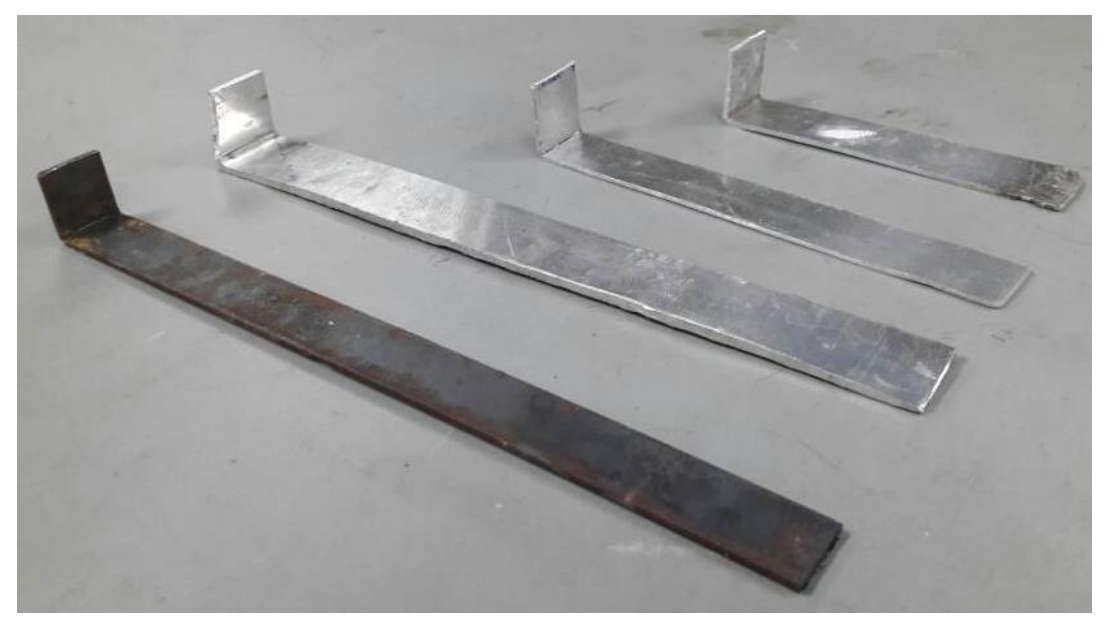

Fig. 4: Steel and aluminium L bracket.

\section{Result and discussion}

Based on table 1 it shows the average result of each experiment. For experiments design $\mathrm{C}$, $\mathrm{D}$ and $\mathrm{E}$, the power are zero because no resistor is connected to the experiment. Resistor is only applied to experiment design E4 and not applied to the others is because based on the result, E4 experiment generate the highest voltage which is $822.71 \mathrm{mV}$. four different resistors are applied to E4 to observe the changes of the current by the TEG. Based on the observation it is shows that the current generated by the TEG is indirectly perpendicular to the resistant of the resistor. Table 1 also shown that the design E4 experiment with $1 \Omega$ resistor produce the highest power with approximately $0.1 \mathrm{~W}$. In order to increase the generated power, TEG can be connect series with another 4 TEGs. 
Table 1. Experiment result based on each model.

\begin{tabular}{|c|c|c|c|c|c|c|c|c|}
\hline Design & $\mathrm{mV}$ & $\mathrm{mA}$ & $\mathrm{P}(\mathrm{W})$ & $\mathrm{Th}$ & $\mathrm{Tc}$ & $\mathrm{dt}$ & $\mathrm{ZT}$ & Efficiency \\
\hline $\mathrm{A}(5 \mathrm{TEG})$ & 40.65 & 8.55 & 0.0003 & 148.4 & 134.5 & 13.9 & 0.0326 & 0.0008 \\
\hline $\mathrm{B}(5 \mathrm{TEG})$ & 100.54 & 7.69 & 0.0008 & 271.3 & 242 & 29.3 & 0.0688 & 0.0028 \\
\hline $\mathrm{C}$ & 28.64 & 0 & 0.0000 & 66.66 & 39.99 & 26.67 & 0.0626 & 0.0076 \\
\hline $\mathrm{D}(15 \mathrm{~cm})$ & 7.23 & 0 & 0.0000 & 37.39 & 27.49 & 9.9 & 0.0232 & 0.0018 \\
\hline $\mathrm{D}(10 \mathrm{~cm})$ & 12.49 & 0 & 0.0000 & 45.75 & 32.36 & 13.39 & 0.0314 & 0.0026 \\
\hline $\mathrm{D}(5 \mathrm{~cm})$ & 17.02 & 0 & 0.0000 & 59.59 & 34.22 & 25.37 & 0.0595 & 0.0078 \\
\hline $\mathrm{D}(2.5 \mathrm{~cm})$ & 79.55 & 0 & 0.0000 & 126.7 & 37.25 & 89.45 & 0.2099 & 0.0506 \\
\hline $\mathrm{E} 1(\mathrm{steel})$ & 25.09 & 0 & 0.0000 & 80.33 & 34.47 & 45.86 & 0.1076 & 0.0202 \\
\hline $\mathrm{E} 2(30 \mathrm{~cm} \mathrm{Al})$ & 258.63 & 0 & 0.0000 & 100.98 & 35.51 & 65.47 & 0.1536 & 0.0337 \\
\hline $\mathrm{E} 3(20 \mathrm{~cm} \mathrm{Al})$ & 409.12 & 0 & 0.0000 & 113.12 & 37.59 & 75.53 & 0.1772 & 0.0400 \\
\hline $\mathrm{E} 4(15 \mathrm{~cm} \mathrm{Al})$ & 822.71 & 0 & 0.0000 & 228.52 & 46.59 & 181.93 & 0.4269 & 0.1108 \\
\hline $\mathrm{E} 4(10 \Omega)$ & 891.81 & 53.091 & 0.0473 & 212.64 & 44.64 & 168 & 0.3942 & 0.1027 \\
\hline $\mathrm{E} 4(7.5 \Omega)$ & 935.13 & 59.645 & 0.0558 & 196.9 & 39.95 & 156.95 & 0.3683 & 0.0986 \\
\hline $\mathrm{E} 4(2.2 \Omega)$ & 916.71 & 63.954 & 0.0586 & 195.72 & 46.41 & 149.31 & 0.3504 & 0.0884 \\
\hline $\mathrm{E} 4(1 \Omega)$ & 883.68 & 112.16 & 0.0991 & 254.76 & 44.93 & 209.83 & 0.4924 & 0.1306 \\
\hline
\end{tabular}

For experiment design $\mathrm{A}$ it shows that the setup was inefficient since the voltage generated is relatively low with small temperature difference. During the experiment, the voltage of the TEG is keep fluctuating up and down rapidly. This show the connection is unstable and it only generate a very small current which is not worth the investment. Meanwhile, for design B experiment is more suitable to utilize for the superior quality TEG since the temperature in both side of the TEG is too high. During the experiment, the soldering flux on the TEG cables were melted and spoiled the device after the first experiment.

In experiment design $C$ the result is also not satisfy since the average voltage result is low. This is because the TEG is located too far from the heat source and the convection 
heat transfer is tend to spread to environment instead of absorb by TEG. While, if the TEG placed nearer to the heat source this will result the big aluminium heat sink that supposed to reject heat from the TEG to act inversely. Meanwhile, experiment design D is done with testing 4 difference distances. In this experiment it shows that distance of $2.5 \mathrm{~cm}$ show the best result. However, the hot temperature of the TEG is still consider low since the average temperature is only half from the heat source temperature which is $126.7^{\circ} \mathrm{C}$.

The last design is created in order to allow the TEG hot side can reach approximately $>200{ }^{\circ} \mathrm{C}$. Initially $30 \mathrm{~cm}$ steel and aluminium L bracket are tested to compare which material provide a better result based on the voltage. Since aluminium has higher heat conductivity, 3 difference size of it is created. The longer the aluminium bracket, the greater heat loss will be occurred due to convection heat transfer. Therefore, shorter aluminium $\mathrm{L}$ bracket is preferable. Not only have higher conduction heat transfer, but shorter L bracket also allow the TEG to get closer to the heat source where convection and radiation heat transfers is higher. To prevent the heat to transfer to the big aluminium heatsink, calcium silicate is place as the TEG holder as shown if Figure 3. The shortest aluminium bracket is chosen as the final design since the result is quite satisfying and able to produce both average closed and close circuit voltage $0.8 \mathrm{~V}$ with $0.12 \mathrm{~A}$ in closed circuit current.

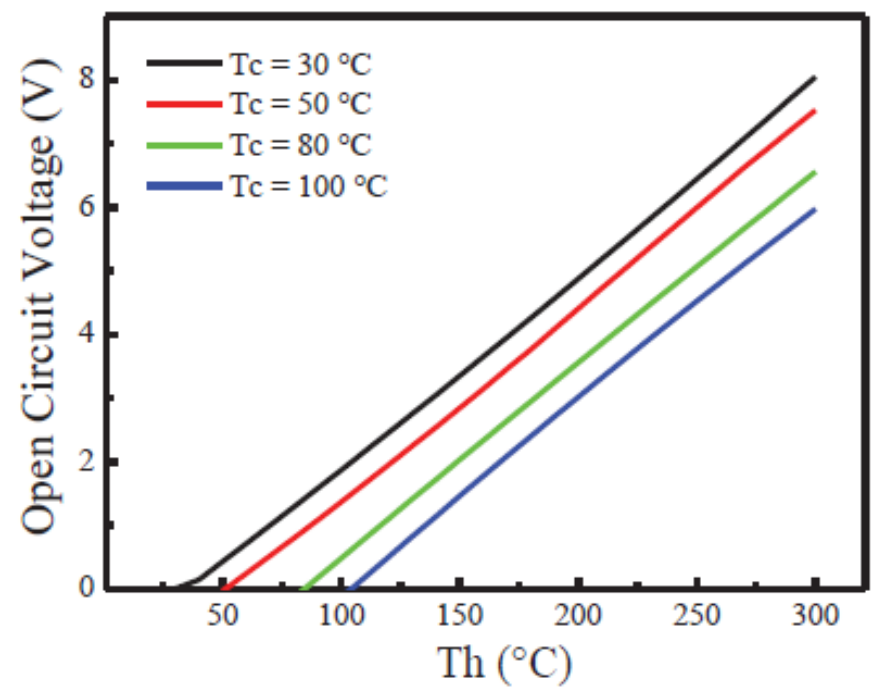

Fig. 5. Chart for open circuit voltage versus Th under various Tc. 


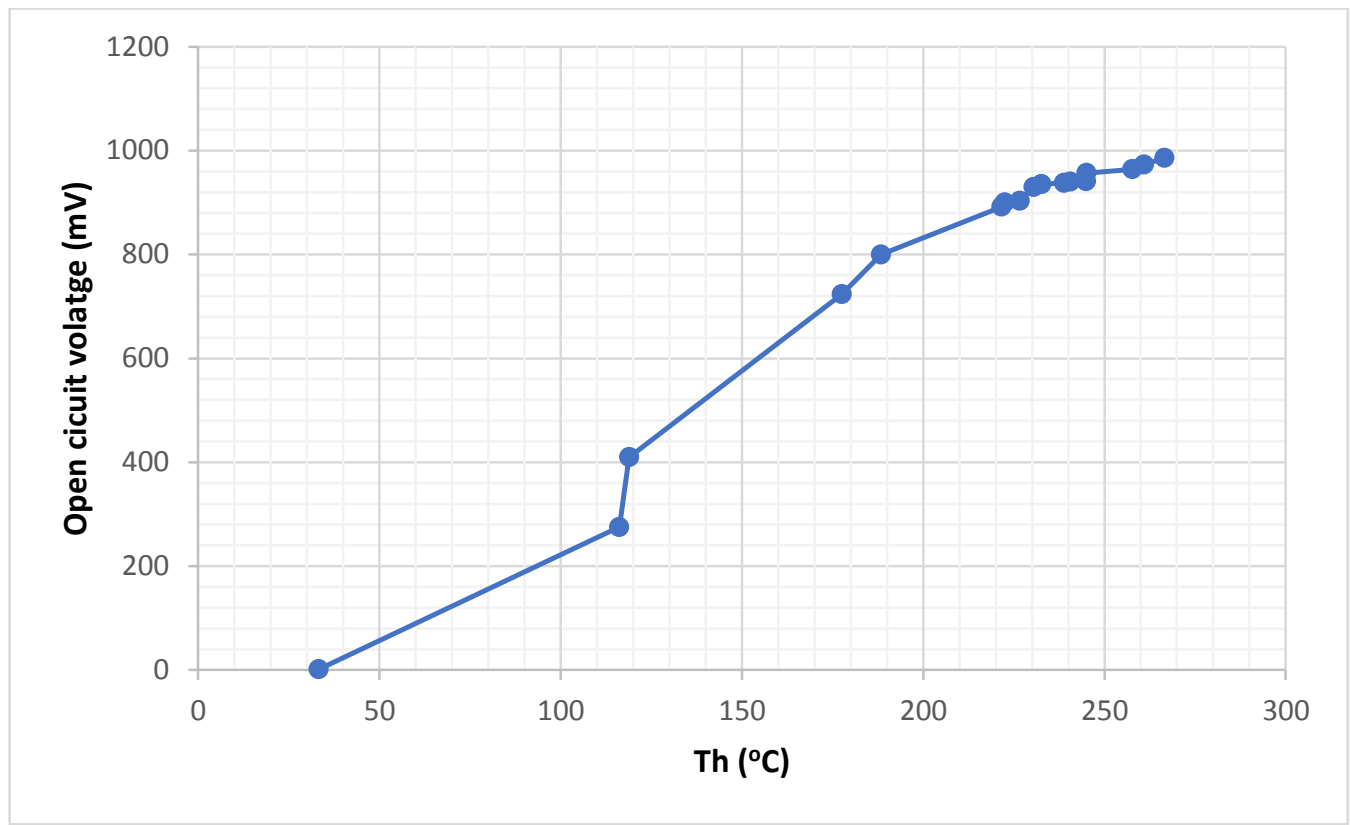

Fig. 6. Experiment chart for open circuit voltage versus Th under Tc $50{ }^{\circ} \mathrm{C}$.

Figure 5 it shows the performance of the high performance TEG based on the provided device's data sheet. Since the average of the Tc of the experiment is approximately $45^{\circ} \mathrm{C}$, the result will be compare to the red colour line where the temperature of $\mathrm{Tc}$ is $50{ }^{\circ} \mathrm{C}$. Meanwhile in Figure 6 shows the result of the Voltage generated with average Tc $45^{\circ} \mathrm{C}$. in the experiment result it shows that the voltage generated is increase rapidly when hot side start reaches temperature $100{ }^{\circ} \mathrm{C}$ to $220^{\circ} \mathrm{C}$ this is because the temperature difference is also increase rapidly. While, the voltage only increases slightly when temperature reach $220{ }^{\circ} \mathrm{C}$ and above since the temperature of the heat supply is only $270{ }^{\circ} \mathrm{C}$ and with effect of external factor such as environment temperature, the temperature on the hot side is only fluctuating between $230{ }^{\circ} \mathrm{C}$ to $260{ }^{\circ} \mathrm{C}$. Based on the comparison between two graphs, it shows that the voltage that generated in the experiment is relatively low even the temperature $\mathrm{Th}$ is follow the temperature from the data sheet. Several factors are stated as the factor that cause the changes such as inconstant Tc during the experiment, fluctuate Th during experiment and environment condition. In order to investigate another factor, more experiment will be conducted to the future. Table 1 shows that the maximum power that can reach by the TEG is only $0.1 \mathrm{~W}$ in design E4. The voltage of open circuit E4 and closed circuit $1 \Omega \mathrm{E} 4$ still remain the same, this mean that this circuit still able to undergo smaller resistant. 0.5 and $0.2 \Omega$ resistant will be tested in the future to observe the changes and 5 TEGs in series closed circuit also will be tested to increase the power. It is expected by combining 5 TEGs into Series closed circuit with smaller resistant, the result will be better and worth the investment since less maintenance is require and it is long last technology.

\section{Conclusion}

In conclusion, TEG technology is consider as green technology since this technology have no moving part, long last and require less maintenance. The temperature differences 
between hot side and cold side of the TEG has become the main parameter in order to generate electricity. Generated energy from the TEG is directed perpendicular with the temperature difference. The higher the temperature differences so do the electric generated. To maximize the efficiency of the power generated, all type of heat transfer which are conduction, convection and radiation is apply to the latest design. While, only conduction cooling with aluminium heat sink was applied to reduce to ease the installation and usage. Design E4 produce the best result in open circuit with voltage $822.71 \mathrm{mV}$. Thus, this design is continued to close circuit with using four difference resistors. Based on the result, design E4 with $1 \Omega$ resistor produce the best result with a power of around $0.1 \mathrm{~W}$.

\section{References}

[1] P. Gokhale, B. Loganathan, J. Crowe, A. Date, and A. Date, Energy Procedia, 110 (2017)

[2] D. Li, J.C. Li, X.Y. Qin, J. Zhang, H.X. Xin, C.J. Song, L. Wang, Energy, 116 (2016)

[3] X. Zhang and L.-D. Zhao, J. Mater., 1, 2 (2015)

[4] D. Champier, Energy Convers. Manag., 140 (2017)

[5] R. Y. Nuwayhid, A. Shihadeh, and N. Ghaddar, Energy Convers. Manag., 46 (2005)

[6] M. Otake, K. Sato, O. Sugiyama, and S. Kaneko, Solid State Ionics, 172 (2004)

[7] H. Pang, Y. Y. Piao, Y. Q. Tan, G. Y. Jiang, J. H. Wang, and Z. M. Li, Mater. Lett., 107 (2013)

[8] L.D. Zhao, C. Chang, G. Tan, M.G. Kanatzidis, Energy \& Env. Sci. (2016)

[9] Y.-L. Pei et al., NPG Asia Mater., 5 (2013) 